\title{
Tibial Tunnel Cyst Formation after Anterior Cruciate Ligament Reconstruction Using a Non-Bioabsorbable Interference Screw
}

\author{
Yogesh V. Joshi, FRCS ${ }^{1}$, Deepu Bhaskar, MS ${ }^{2}$, Padmanabh M. Phaltankar, FRCS ${ }^{1}$, and Charalambos P. \\ Charalambous, FRCS $^{2-4}$ \\ ${ }^{1}$ Department of Trauma and Orthopaedics, North Manchester General Hospital, Manchester; ${ }^{2}$ Department of Trauma and Orthopaedics, Blackpool Victoria Hospital, \\ Blackpool; ${ }^{3}$ School of Medicine and Dentistry, University of Central Lancashire, Preston; ${ }^{4}$ Institute of Inflammation and Repair, Faculty of Medical and Human Sciences, \\ University of Manchester, Manchester, UK
}

\begin{abstract}
Tibial cyst formation following the use of bioabsorbable interference screws in anterior cruciate ligament (ACL) reconstruction is well-described; however, cyst formation after the use of metallic interference screws is not well-documented. We describe a case of osteolytic lesion of the proximal tibia presenting to us 20 years after ACL reconstruction using an autologous bone-tendon-bone graft. The original graft fixation technique was interference fixation with a metal screw in the tibial and femoral tunnels. A two-stage revision reconstruction of the ACL was undertaken with curettage and bone grafting of the tibial lesion in the first stage and reconstruction using a four-strand hamstring tendon in the second stage. The patient recovered satisfactorily with complete healing of the cyst and returned to pre-injury level of activities. We have reviewed case reports and case series that describe the aetiology of intra-osseous cyst formation following ACL reconstruction.
\end{abstract}

Keywords: Knee, Tibial cyst, Anterior cruciate ligament reconstruction, Interference screw, Metal

Anterior cruciate ligament (ACL) reconstructions using allografts as well as autografts have been associated with intra- as well as extra-osseous cyst formation ${ }^{1-9)}$. In the case of allografts, incomplete incorporation of the graft and foreign body reaction associated with the allograft itself have been implicated ${ }^{1)}$. Even following the use of autografts, such as hamstring tendons and bone-tendon-bone grafts, with bioabsorbable interference screw fixation, intra-osseous as well as extra-osseous cysts have been reported. In the latter case, immunological and biological reactions related to the use of bioabsorbable screws have been implicated in

Received November 14, 2014; Revised (1st) January 7, 2015;

(2nd) April 12, 2015; Accepted May 14, 2015

Correspondence to: Deepu Bhaskar, MS

Department of Trauma and Orthopaedics, Blackpool Victoria Hospital, Whinney Heys Road, Blackpool, Lancashire, FY3 8NR, UK

Tel: +44-01253-300000, Fax: +44-01253-300000

E-mail: deepu.bhaskar@gmail.com

This is an Open Access article distributed under the terms of the Creative Commons Attribution Non-Commercial License (http://creativecommons.org/licenses/by-nc/4.0/) which permits unrestricted non-commercial use, distribution, and reproduction in any medium, provided the original work is properly cited. their causation ${ }^{2,3)}$. In contrast, reports of cystic changes associated with the use of metallic interference screws for graft fixation are rare $^{1,4)}$. In the series by Victoroff et al. ${ }^{1)}$ and Simonian et al. ${ }^{4)}$, pretibial cysts were more common than osseous cysts after the use of allografts in ACL reconstruction. Our case describes an intraosseous cyst of proximal tibia in a patient who presented with graft re-rupture 20 years after ACL reconstruction using metallic interference screws for graft fixation.

\section{Case Report}

A 47-year-old man, an ex-gymnast, presented with symptoms of instability and locking of his left knee. He had a few falls recently due to knee instability while participating in recreational sports. There was no significant limitation of functional activity. In the primary care setting, he had a course of physiotherapy with no improvement in symptoms. He reported that he had had a sports-related ACL injury 22 years ago and undergone ACL reconstruction using an autologous bone-patellar tendon-bone graft two years later. 
On clinical examination, he walked without any walking aids and had a well-healed surgical scar on the anterior aspect of the left knee. There was no effusion, the medial joint line was tender and McMurray's test was positive. The range of motion was from 0 to 110 degrees. The Lachman test was positive with absence of a firm end point (grade IIIB), as was the anterior drawer test (grade III).

Radiographs of the knee revealed early degenerative changes in the medial compartment and the patellofemoral joint. The presence of metallic screws in the femur and tibia were demonstrated. There was presence of cystic changes around the tibial screw (Fig. 1). A magnetic resonance imaging (MRI) scan confirmed presence of a complex medial meniscal tear, rupture of the ACL graft and presence of an intra-osseous cyst adjacent to the tibial interference screw with surrounding bone marrow oedema (Fig. 2).

As the patient was symptomatic with recurrent giving way of the knee and only early degenerative changes in the knee, he was offered a two-stage ACL reconstruction. In the first stage, removal of the tibial interference screw, curettage and autologous bone

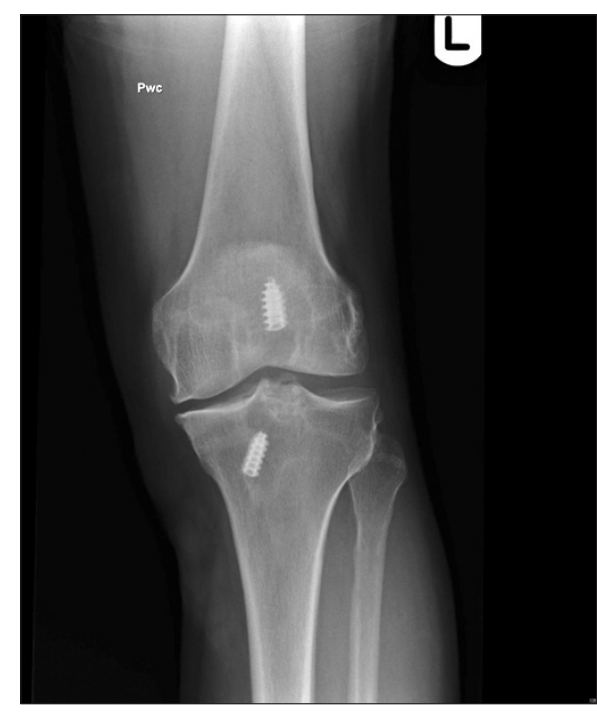

Fig. 1. Knee radiograph at presentation demonstrating cyst formation around the tibial interference screw. L: left.
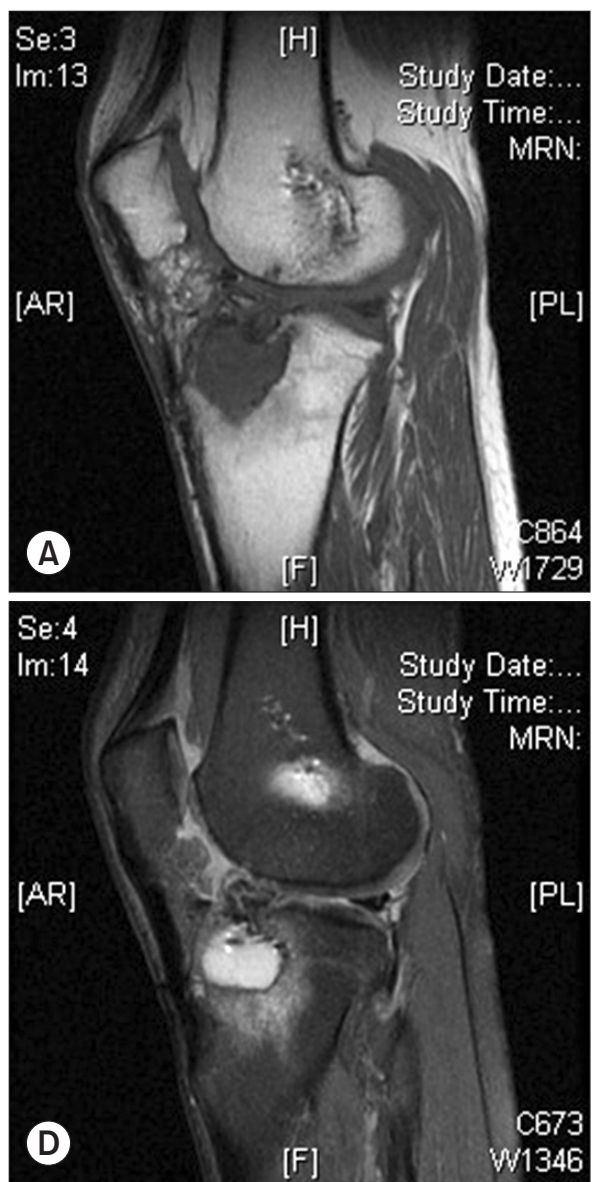
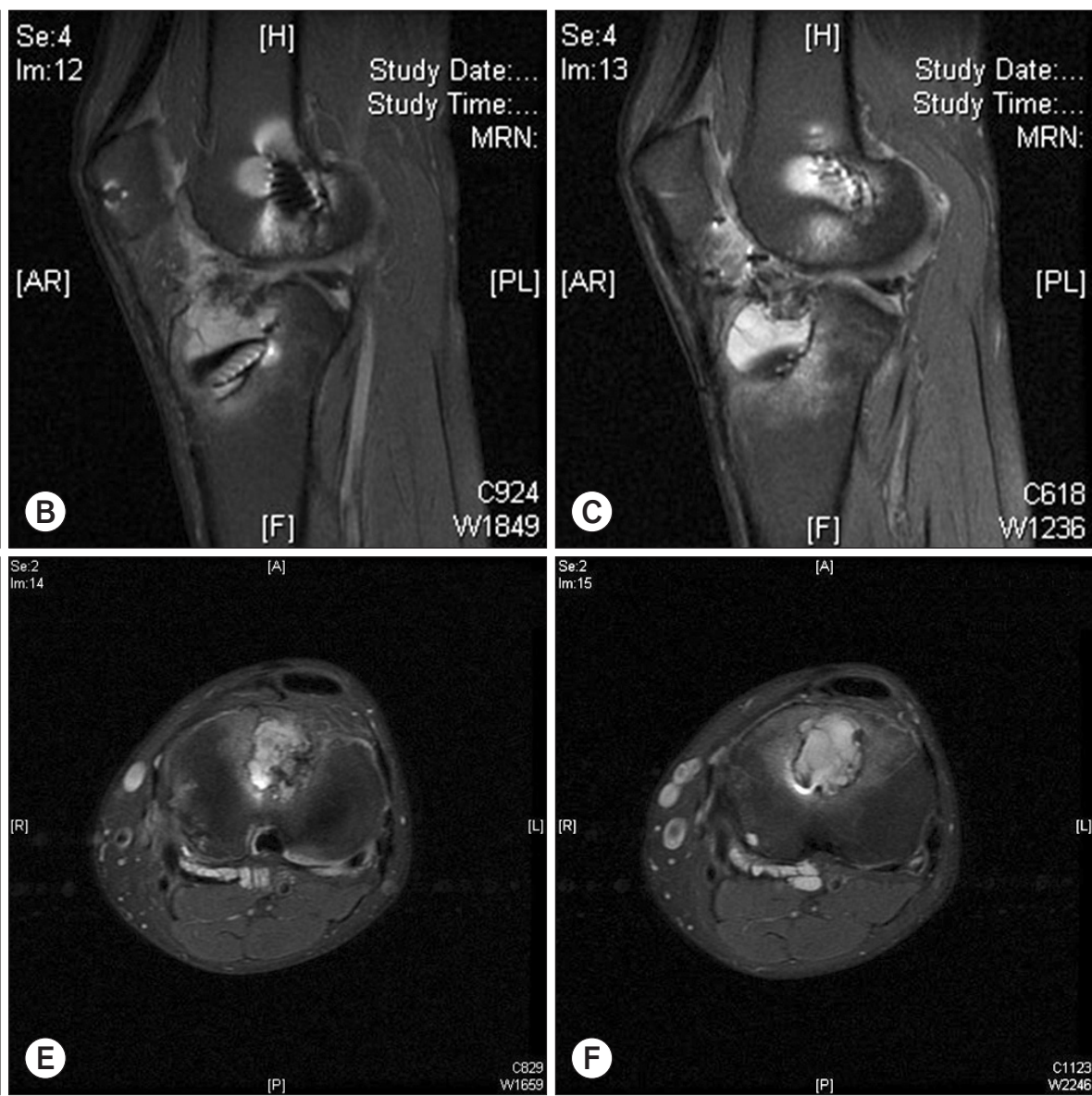

Fig. 2. Preoperative magnetic resonance imaging scans showing cystic changes in the tibia. 

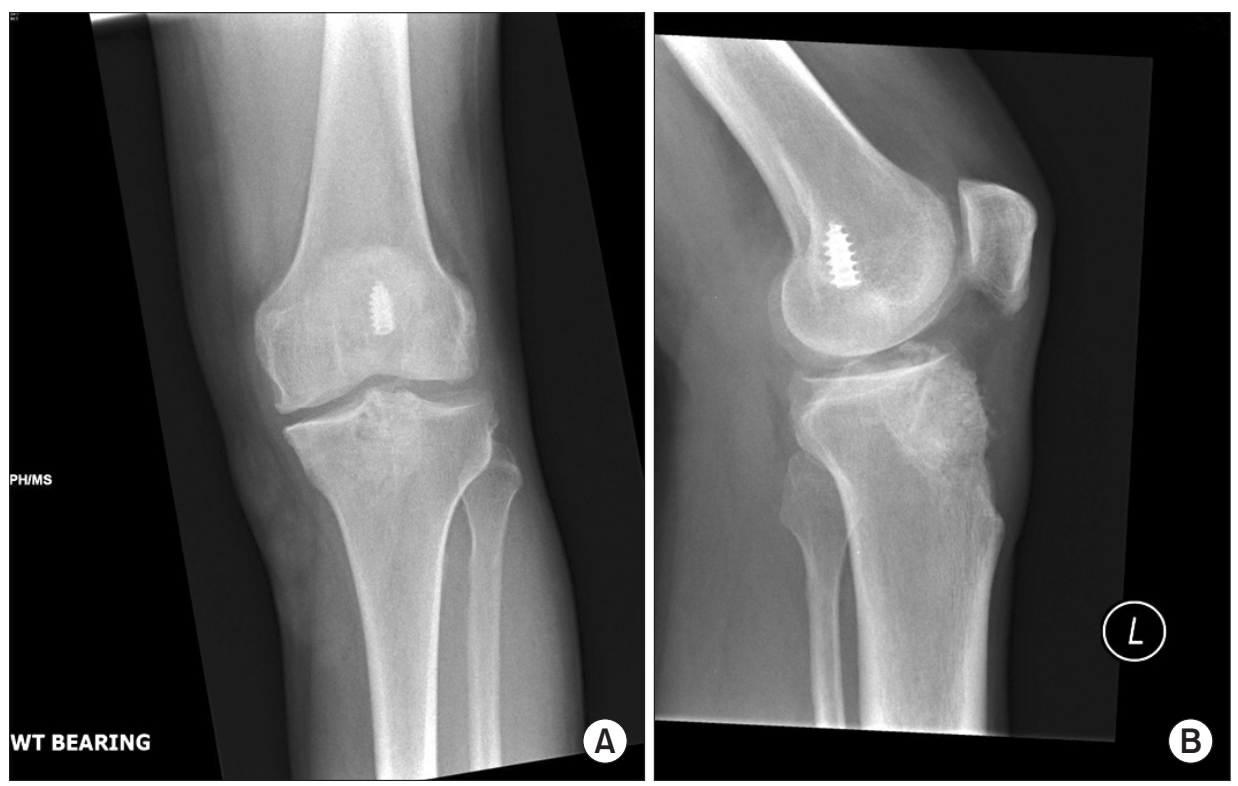

Fig. 3. Anteroposterior (A) and lateral (B) radiographs obtained after the first-stage anterior cruciate ligament reconstruction showing graft incorporation. L: left.
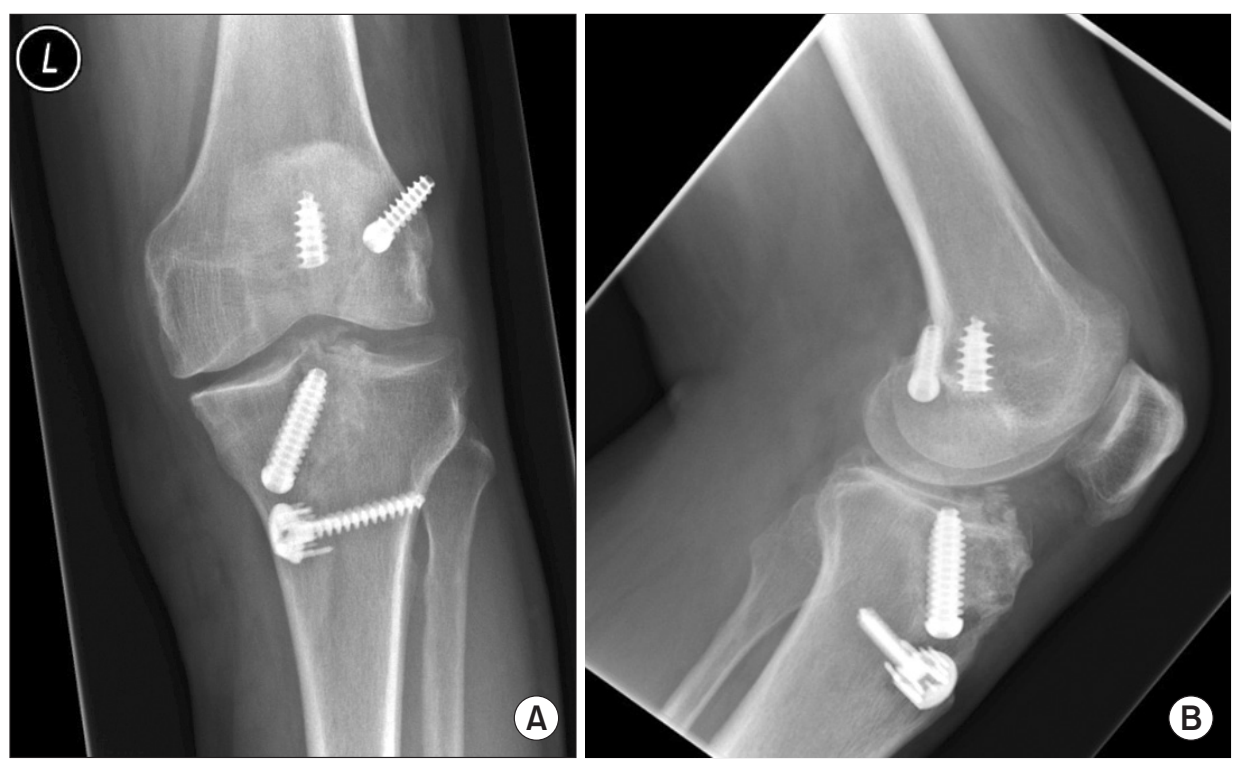

Fig. 4. (A) Anteroposterior radiograph obtained after the second-stage anterior cruciate ligament (ACL) reconstruction. Note dual fixation of the graft in the tibia. (B) Lateral radiograph obtained after the second-stage ACL reconstruction. L: left.

grafting of the cyst were undertaken along with arthroscopic partial medial meniscectomy. There was no demonstrable communication between the cyst and the knee joint or the pretibial soft tissue.

Macroscopically, the tissue obtained from the cyst was fibrous tissue intermingled with bone. Histopathology of the cyst confirmed fibro-connective and fibro-vascular tissue with patchy chronic inflammation. There were no macrophages present to suggest a foreign body reaction.

Four months following the bone grafting, radiographic cyst healing was demonstrated (Fig. 3), and a second-stage ACL re- construction was performed using an ipsilateral autologous fourstrand hamstring graft, which was followed by fixation using an interference screw on the femoral side and dual fixation using an interference screw and a spiked washer on the tibial side (Fig. 4). The patient had an uneventful recovery from both procedures. At the six months follow-up, the patient had no symptoms of instability. Clinical examination demonstrated stable knee with normal Lachmann and anterior drawer tests (grade I). Radiographs showed healed tibial cyst and good position of the implants. 


\section{Discussion}

A variety of mechanisms have been postulated for tunnel enlargement and cyst formation following ACL reconstruction. They include foreign body reaction to bioabsorbable screws ${ }^{3)}$, breakdown of bio-absorbable interference screws ${ }^{5}$, leakage of joint fluid through the tunnel ${ }^{6}$ often due to the graft-tunnel diameter mismatch $^{4)}$, eccentric placement of a graft, intra-osseous graft necrosis with incomplete graft incorporation ${ }^{1)}$ and graft micro-motion ${ }^{1,45)}$. However, the phenomenon of intra-osseous cyst formation remains poorly understood. There have been many case reports describing cystic reaction following the use of bioabsorbable implants in ACL reconstruction ${ }^{1,3-6)}$. In 2009, Konan and Haddad $^{2)}$ published a review on the adverse effects of the use of bioabsorbable interference screws in ACL reconstruction. Warden et al. ${ }^{7.8)}$ published MRI follow-up results at 2 years and 10 years, respectively, after ACL reconstruction using bioabsorbable interference screws in 20 knees: all 6 patients available at the 10year follow-up had fluid collection in the tibial tunnel and 4 out of the 6 had fluid in the femoral tunnel.

Since introduced by Lambert in 1983 and popularized by Kurosaka and colleagues ${ }^{9)}$, stainless steel and titanium interference screws have been in use in ACL reconstruction. Compared to the use of bioabsorbable screws, cyst formation associated with metallic screws seems to be a rare entity ${ }^{1,4)}$. Stainless steel screws become surrounded by a fibrous layer rich in inflammatory cells. However, titanium, unlike other metals that do not integrate well with surrounding bones, forms an oxide layer on which calcium and phosphate precipitate and osteoblasts can bind and thus integrate directly to bone with minimal inflammatory response ${ }^{10)}$. In spite of the theoretical advantages of titanium screws over stainless steel ones, there are not many reports of cysts associated with either type of metallic screws.

Our case describes cyst formation in the tibial tunnel associated with the use of a metallic interference screw in ACL reconstruction. An inflammatory origin for the cyst was suggested by histopathology. However, titanium is expected to be an inert material. Moreover, the lack of inflammatory reaction or cyst formation around the femoral interference screw goes against inflammatory reaction against the screw as a cause of cyst formation in this case. In a case series where metallic screws were associated with cyst formation, Victoroff et al. ${ }^{1)}$ postulated that incomplete incorporation of the allograft tissue within the bone tunnels or pressure necrosis of the graft fibres was the cause of cyst development. However, in our case, an autologous graft was used in the primary surgery. Simonian et al. ${ }^{4)}$ reported one case of pretibial cyst (not tibial bone cyst) in the knee with a metallic screw and autologous graft; however, in his case, there was communication with the joint, and joint fluid leak was proposed as the cause of cyst formation. The same study suggested graft micro-motion as the cause of late development of the cyst, which seems a plausible explanation for cyst formation in cases where an inert metal and autologous graft are used for fixation.

The management of such cases are complicated by the presence of cyst and the need to treat ACL deficiency. Investigation in the form of computed tomography or MRI scanning helps to determine the precise location of previous tunnels and to plan placement of future tunnels. In our case, we used a two- stage procedure. In the first stage, the cyst can be treated by making a window in the tibia and curetting the cyst with bone grafting. In the second stage of ACL reconstruction, the placement of the tunnels should be made in anatomical locations, and fixation should be stable and rigid enough to prevent any future complications. In our patient, we combined both types of fixations for the final graft i.e. suspensory as well as aperture fixation. It was postulated that such fixation would prevent any mobility of the graft in the tunnel, especially in revision surgery.

Our case report describes cyst formation in the proximal tibia following metallic interference screw fixation of an ACL reconstruction graft. We think that the most likely mechanism for osseous cyst formation in the knee with a non-bioabsorbable screw and an autologous graft would be micro-motion of the graft within the tunnel, and a two-stage procedure has been proposed as a treatment option in this complex situation. This case report adds to the few reported cases where cyst formation was associated with metallic interference screw usage.

\section{Conflict of Interest}

No potential conflict of interest relevant to this article was reported.

\section{References}

1. Victoroff BN, Paulos L, Beck C, Goodfellow DB. Subcutaneous pretibial cyst formation associated with anterior cruciate ligament allografts: a report of four cases and literature review. Arthroscopy. 1995;11:486-94.

2. Konan S, Haddad FS. A clinical review of bioabsorbable interference screws and their adverse effects in anterior cruciate ligament reconstruction surgery. Knee. 2009;16:6-13.

3. Gonzalez-Lomas G, Cassilly RT, Remotti F, Levine WN. Is 
the etiology of pretibial cyst formation after absorbable interference screw use related to a foreign body reaction? Clin Orthop Relat Res. 2011;469:1082-8.

4. Simonian PT, Wickiewicz TL, O’Brien SJ, Dines JS, Schatz JA, Warren RF. Pretibial cyst formation after anterior cruciate ligament surgery with soft tissue autografts. Arthroscopy. 1998;14:215-20.

5. Tsuda E, Ishibashi Y, Tazawa K, Sato H, Kusumi T, Toh S. Pretibial cyst formation after anterior cruciate ligament reconstruction with a hamstring tendon autograft. Arthroscopy. 2006;22:691.

6. Thaunat M, Chambat P. Pretibial ganglion-like cyst formation after anterior cruciate ligament reconstruction: a consequence of the incomplete bony integration of the graft? Knee Surg Sports Traumatol Arthrosc. 2007;15:522-4.
7. Warden WH, Friedman R, Teresi LM, Jackson DW. Magnetic resonance imaging of bioabsorbale polylactic acid interference screws during the first 2 years after anterior cruciate ligament reconstruction. Arthroscopy. 1999;15:474-80.

8. Warden WH, Chooljian D, Jackson DW. Ten-year magnetic resonance imaging follow-up of bioabsorbable poly-L-lactic acid interference screws after anterior cruciate ligament reconstruction. Arthroscopy. 2008;24:370.

9. Schindler OS. Surgery for anterior cruciate ligament deficiency: a historical perspective. Knee Surg Sports Traumatol Arthrosc. 2012;20:5-47.

10. Suchenski M, McCarthy MB, Chowaniec D, Hansen D, McKinnon W, Apostolakos J, Arciero R, Mazzocca AD. Material properties and composition of soft-tissue fixation. Arthroscopy. 2010;26:821-31. 